\title{
Quantum Fluctuations and the Exchange Bias Field
}

\author{
G.J. Mata and E. Pestana \\ Departamento de Física, Universidad Simón Bolivar, \\ Apartado 89000, Caracas 1080A, Venezuela \\ Miguel Kiwi \\ Facultad de Física, Pontificia Universidad Católica de Chile, Casilla 306, Santiago 22, Chile \\ Hugues Dreysse \\ IPCMS - GEMME, 23, rue du Loess BP43, 67034 Strasbourg Cedex 2 France
}

(Dated: November 20, 2018)

\begin{abstract}
Ground-state fluctuations reduce the zero-temperature magnetic moments of the spins in a quantum antiferromagnet. In the neighborhood of surfaces, interfaces, and other defects which break translational symmetry, these fluctuations are not uniform. Because of this the magnetic moments of up and down spins do not exactly compensate each other - as they do in a bulk antiferromagnet. At a surface or interface this leads to a small magnetic dipole density. The corresponding dipole field can account for the magnitude of observed exchange fields. At finite temperatures localized surface (interface) excitations are populated and change the dipole density. This gives rise to the temperature-dependence of the exchange field. We expect the fluctuation-induced surface dipole density to play a role in the magnetic properties of antiferromagnetic nano-particles, as well.
\end{abstract}


Exchange anisotropy arises when two differently ordered magnetic materials, which are in contact, are cooled in an external magnetic field through their ordering temperatures. It has been observed, for example, in clusters or small particles, ferromagnetic (FM) films deposited on single-crystal or polycrystalline antiferromagnetic (AF) substrates, FM/AF thin film bilayers, and spin glasses. In these systems the center of the hysteresis loop is shifted by an amount called the exchange bias field. With the convention that the positive field direction is that of the cooling field, this exchange bias is, in most cases, negative.

Although exchange anisotropy has attracted the attention of physicists and materials scientists for almost half a century [1, 2, 3, 4] and has resulted in extensive technological applications in the storage and sensor industries [5], a full understanding of its physical origin has not been achieved.

From the experimental results, it is now certain that the effect is due to a fixed spin arrangement on the antiferromagnetic side of the interface [6, 7, 8]. However, the nature of this arrangement, and the microscopic mechanism leading to the exhange bias field are still open questions. Most of the theoretical work has made use of the classical Heisenberg model in various forms [4], an exception being the contribution of Suhl and Schuller [9], who have interpreted the exchange field as a self-energy shift due to the emission and reabsorption of antiferromagnetic spin waves. Recently, a theory based on the Dzyaloshinsky-Moriya interaction has been put forward by Ijiry et al [10].

In this letter we argue that quantum fluctuations lead to a two-dimensional dipole moment density at the ferromagnetic-antiferromagnetic interface. The magnetic field due to this dipole density can explain exchange bias.

Why are quantum fluctuations relevant to the physics of antiferromagnets? Let us briefly consider this question in the case of a two-sublattice antiferromagnet. The order parameter relevant here is the staggered magnetization: $<\hat{\mathbf{M}}_{A F}>=g \mu_{B}<\sum_{\alpha} \hat{\mathbf{S}}_{\alpha}-\sum_{\beta} \hat{\mathbf{S}}_{\beta}>$, where $\hat{\mathbf{S}}_{\alpha}$ and $\hat{\mathbf{S}}_{\beta}$ denote the spin operators at the spin-up and spin-down sub-lattices. If we assume a Heisenberg Hamiltonian $\mathcal{H}=\sum_{i j} J_{i j} \hat{\mathbf{S}}_{i} \cdot \hat{\mathbf{S}}_{j}$, we can see that $\left[\hat{\mathbf{M}}_{A F}, \mathcal{H}\right] \neq 0$, in other words, that $\hat{\mathbf{M}}_{A F}$ is not constant in time. Therefore its time-averaged value must be smaller than its maximum value, even at $T=0$. (If $N$ is the number of spins and $\sqrt{S(S+1)}$ is the spin magnitude, this maximum value is $N S$.) In a translationally invariant system the reduction of the magnetization is equally shared by all spins. Moreover, since the spin up and spin down sublattices are equivalent then $\sum_{\alpha} \hat{\mathbf{S}}_{\alpha}=-\sum_{\beta} \hat{\mathbf{S}}_{\beta}$ - that is, the system magnetic 
moment is zero. But when translational symmetry is broken by surfaces, interfaces, or other defects, a finite magnetic moment can appear because the decrease of spin-up averages need not equal the corresponding spin-down reduction [11, 12, 13]. In the calculations outlined below we find that the magnitude of this magnetic moment is only a few percent of that of a fully uncompensated spin, which is consistent with the experimental results.

Let us now focus on a specific model, in which atomic spins of magnitude $\sqrt{S(S+1)}$ are located at the sites of a bcc lattice. This system is assumed to be divided in two halves by a (001) interface. To the left of the interface, nearest neighbor spins are coupled ferromagnetically by the exchange integral $-J_{F}$. To the right, nearest neighbor spins are coupled antiferromagnetically by the exchange integral $J_{A}$. Across the interface, spins are coupled by the exchange integral $-J_{0}$. Here we examine three cases, with $J_{0}<0, J_{0}>0$, and $J_{0}=0$, respectively. The latter corresponds to a free antiferromagnetic surface.

We decompose the bcc lattice into planes parallel to the interface. In the antiferromagnetic side, each of these planes is ferromagnetic and its spin direction alternates from one plane to the next; we group these planes in pairs an label each pair with the index $l \geq 0$, in such a way that $l=0$ labels the pair of planes closest to the interface; for each pair we label the corresponding planes with the subscript $\alpha$ for spins up, and the label $\beta$ for spins down. In the ferromagnetic side each index $(l<0)$ denotes a single, spin up, layer. This choice of notation reflects the fact that in the antiferromagnet the unit cell is doubled. The Hamiltonian can be written as:

$$
\begin{aligned}
\mathcal{H}= & \sum_{l=0}^{+\infty} \sum_{\mathbf{R}, \delta}\left[J_{l, l} \mathbf{S}_{\alpha}(l, \mathbf{R}) \cdot \mathbf{S}_{\beta}(l, \mathbf{R}+\delta)+J_{l, l+1} \mathbf{S}_{\beta}(l, \mathbf{R}+\delta) \cdot \mathbf{S}_{\alpha}(l+1, \mathbf{R})\right]+ \\
& \sum_{l=-\infty}^{-1} \sum_{\mathbf{R}, \delta} J_{l, l+1} \mathbf{S}_{\alpha}(l, \mathbf{R}+\delta) \cdot \mathbf{S}_{\alpha}(l+1, \mathbf{R}),
\end{aligned}
$$

where $\mathbf{R}=a\left(n_{1} \hat{\mathbf{x}}+n_{2} \hat{\mathbf{y}}\right)$ is a two-dimensional lattice point, $a$ is the lattice constant, $n_{1}$ and $n_{2}$ are integers, $\delta=a( \pm \hat{\mathbf{x}}+ \pm \hat{\mathbf{y}})$, and $\mathbf{S}_{a}(l, \mathbf{R})\left[\mathbf{S}_{b}(l, \mathbf{R})\right]$ is a spin in the $\alpha(\beta)$ plane of the l-th pair (and at site $\mathbf{R}$ in that plane).

We now use the Holstein-Primakoff transformation to rewrite the Hamiltonian in terms of boson operators $a$ and $b$ :

$$
S_{a}^{z}(l, \mathbf{R})=S-a^{\dagger}(l, \mathbf{R}) a(l, \mathbf{R})
$$




$$
\begin{gathered}
S_{a}^{+}(l, \mathbf{R})=\left[2 S-a^{\dagger}(l, \mathbf{R}) a(l, \mathbf{R})\right]^{1 / 2} a(l, \mathbf{R}), \\
S_{b}^{z}(l, \mathbf{R})=-S+b^{\dagger}(l, \mathbf{R}) b(l, \mathbf{R}),
\end{gathered}
$$

and

$$
S_{b}^{-}(l, \mathbf{R})=\left[2 S-b^{\dagger}(l, \mathbf{R}) b(l, \mathbf{R})\right]^{1 / 2} b(l, \mathbf{R}),
$$

We neglect spin wave interactions and therefore we discard quartic and higher order terms. To take advantage of the translational symmetry we define:

$$
a(l, \mathbf{k})=\frac{1}{\sqrt{N}} \sum_{\mathbf{R}} a(l, \mathbf{R}) \exp (\mathrm{ik} \cdot \mathbf{R})
$$

and

$$
b(l, \mathbf{k})=\frac{1}{\sqrt{N}} \sum_{\mathbf{R}} b(l, \mathbf{R}) \exp (-\mathrm{ik} \cdot \mathbf{R}) .
$$

The last transformation separates the Hamiltonian into a sum of Hamiltonians, one for each value of the two-dimensional wavector $\mathbf{k}$. Each of these Hamiltonians can be thought of as describing a one-dimensional chain, the parameters of which depend on $\mathbf{k}$ only through the function $\gamma_{\mathbf{k}}=\cos \left(k_{x} / 2\right) \cos \left(k_{y} / 2\right)$. Once this separation is made, ground-state properties become sums over k. One-dimensional chains are conveniently analyzed using Green functions, which we now define:

$$
G_{l l^{\prime}}^{a a}=-\frac{\mathrm{i}}{\hbar} \int_{-\infty}^{\infty} d t e^{\mathrm{i} \omega t} \theta(t)\left\langle\left[a(l, \mathbf{k}, t), a^{\dagger}\left(l^{\prime}, \mathbf{k}, 0\right)\right]\right\rangle
$$

where $\langle A\rangle$ denotes the thermal average of $A$ and the operators are in the Heisenberg representation. We define the functions $G_{l l^{\prime}}^{b b}, G_{l l^{\prime}}^{a b}$, and $G_{l l^{\prime}}^{b a}$ in the same fashion. The average spin at plane $l$ is given by

$$
\begin{gathered}
\left\langle S_{l, a}^{z}\right\rangle=S+\sum_{\mathbf{k}} \frac{1}{\pi} \operatorname{Im} \int_{-\infty}^{\infty} d \omega \frac{G_{l l}^{a a}\left(\omega, \gamma_{\mathbf{k}}\right)}{e^{\hbar \omega / k_{B} T}-1} \\
\left\langle S_{l, b}^{z}\right\rangle=-S-\sum_{\mathbf{k}} \frac{1}{\pi} \operatorname{Im} \int_{-\infty}^{\infty} d \omega \frac{G_{l l}^{b b}\left(\omega, \gamma_{\mathbf{k}}\right)}{e^{\hbar \omega / k_{B} T}-1}
\end{gathered}
$$

The spectral distributions $\frac{1}{\pi} \operatorname{Im} G$ in Equations 9 and 10 contain the contributions of interface and bulk excitations. The interface excitations are localized in a few layers about 
interface. They appear for all values of the model parameters and dominate the spin reduction, and hence the net magnetization, at the interface. When $T=0$ the integrands in 9 and 10] are zero for $\omega>0$. The negative $\omega$-axis gives the effect of virtual spin waves on ground-state fluctuations [13]. At finite temperatures real spin waves are excited which further change the net magnetization.

In figures 1 1 and 2 we show the magnetization per unit cell in the antiferromagnetic side of the interface. In both cases, the maximum net magnetization is of the order of a few percent of that of an atomic spin. In figure 1 the coupling across the interface is ferromagnetic and the net magnetization is parallel to the ferromagnetic moments. In figure 2 the coupling across the interface is antiferromagnetic and the net magnetization is antiparallel to that of the ferromagnet. In general, we find that for $\left|J_{0}\right| \lesssim\left|J_{A}\right|$ the net magnetization of the antiferromagnet is of the order of a few percent of that of an atomic spin.

The magnetic moments of the antiferromagnetic spins produce a dipolar magnetic field. This is a long range effect. We can estimate the magnitude of this field assuming that the net magnetization is uniformly distributed at a single layer. If we take this layer to be a disk of radius $R$, the field at a distance $z$ on the axis is given by $B(z)=\left(\mu_{0} g \mu_{B} \delta S / R a^{2}\right)(1+$ $\left.z^{2} / R^{2}\right)^{-3 / 2}$. With $R \sim 1 \mathrm{~nm}, \delta S \sim 10^{-2}$, and the lattice constant $a \sim 0.1 \mathrm{~nm}$, we find that $B(0) \sim 10^{3}$ oersted.

Notice that in figure1 1 the exchange bias field is negative, whereas in figure 2, it is positive.

We now turn our attention to the free surface case, which we can describe by simply taking $J_{0}=0$. In figure 3 we show the low temperature variation of the net surface magnetization. As the temperature rises, surface spin waves are excited which change the dipole density.

The magnetic moment is, again, of the order of a few percent of that of an uncompensated monolayer, as has been observed by Takano et al. 15] in $\mathrm{CoO} / \mathrm{MgO}$ multilayers. For this particular system the sign of the exchange field reverses. Such kind of reversal has been experimentally observed [14].

In summary, we have shown that the dipole field generated by uncompensated quantum fluctuations can account for the observed exchange bias fields. The temperature dependence is explained in terms of the excitation of surface spin waves which, for low enough temperatures, can be accounted for by our linear theory. Finally, since we find that pure antiferromagnets develop a surface magnetic moment, our theory could be tested by experimental studies of clean antiferromagnetic surfaces. 


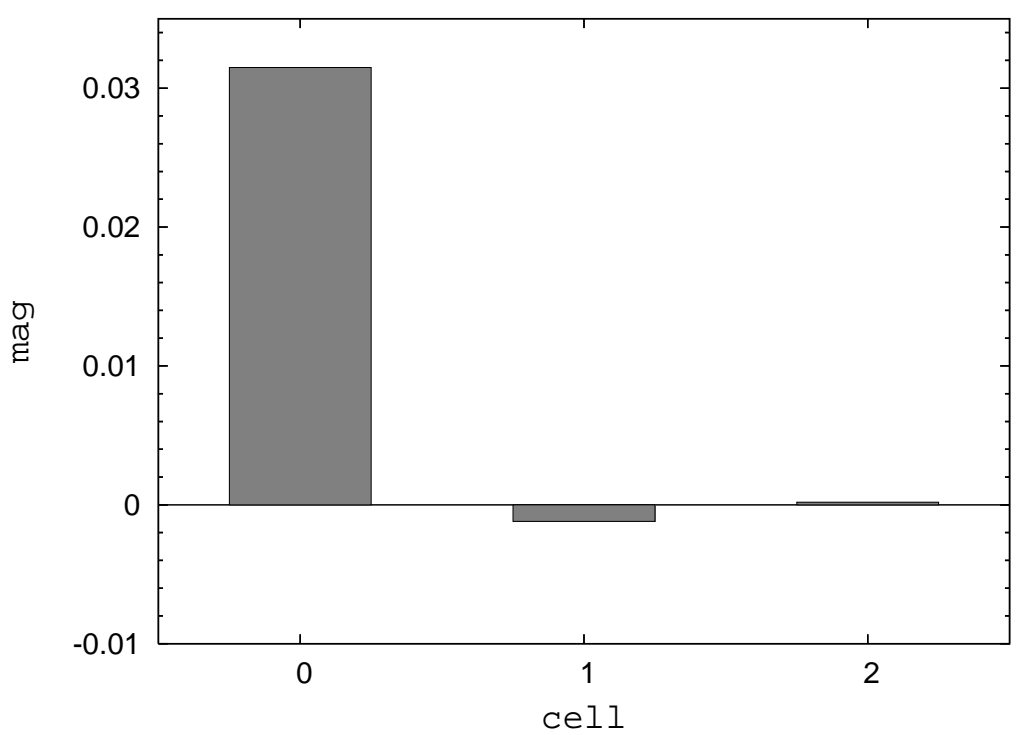

FIG. 1: Net magnetization per unit cell in the antiferromagnetic side of the interface. The index cell denotes a pair of layers of opposite spins. In units such that $J_{A}+J_{F}=1$, the parameters are $J_{A}=0.615, J_{F}=0.385$, and $J_{0}=0.5$.

[1] W.H. Meiklejohn and C.P. Bean, Phys. Rev. 102, 904 (1957).

[2] A.E. Berkowitz and Kentaro Takano, J. Magn. Magn. Mater. 200, 552 (1999).

[3] J. Nogués and Ivan K. Schuller, J. Magn. Magn. Mater. 192, 203 (1999).

[4] M. Kiwi, J. Magn. Magn. Mater. 234, 584 (2001).

[5] B. Dieny et al., Phys. Rev. B 43, 1297 (1991).

[6] H. Ohldag et al., Phys. Rev. Lett. 87, 247201 (2001).

[7] A. Hoffmann et al., Phys. Rev. B 66, 220406 (2002).

[8] H. Ohldag et al., Phys. Rev. Lett. 91, 017203 (2003).

[9] H. Suhl and I.K. Schuller, Phys. Rev. B 58, 258 (1998).

[10] Y. Ijiri et al., (to be published).

[11] G.J. Mata and E. Pestana, in New Trends in Magnetism, Magnetic Materials, and Their Applications, edited by J.L. Morán López and J.M. Sanchez (Plenum, New York, 1994) pp. 201-209.

[12] G.J. Mata and E. Pestana, Phys. Rev. B 42, 885 (1990).

[13] G.J. Mata and E. Pestana, Phys. Rev. B 31, 7285 (1985). 


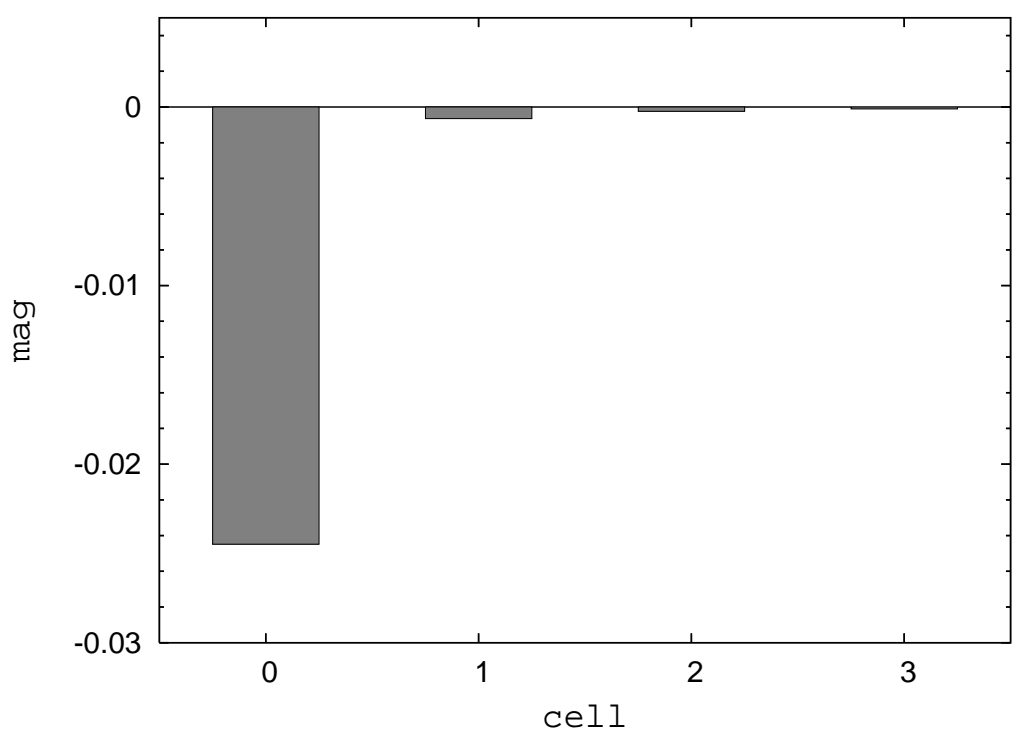

FIG. 2: Net magnetization per unit cell near the antiferromagnetic side of the interface. The index cell denotes a pair of layers of opposite spins. In units such that $J_{A}+J_{F}=1$, the parameters are $J_{A}=-0.17, J_{F}=0.83$, and $J_{0}=0.08$.

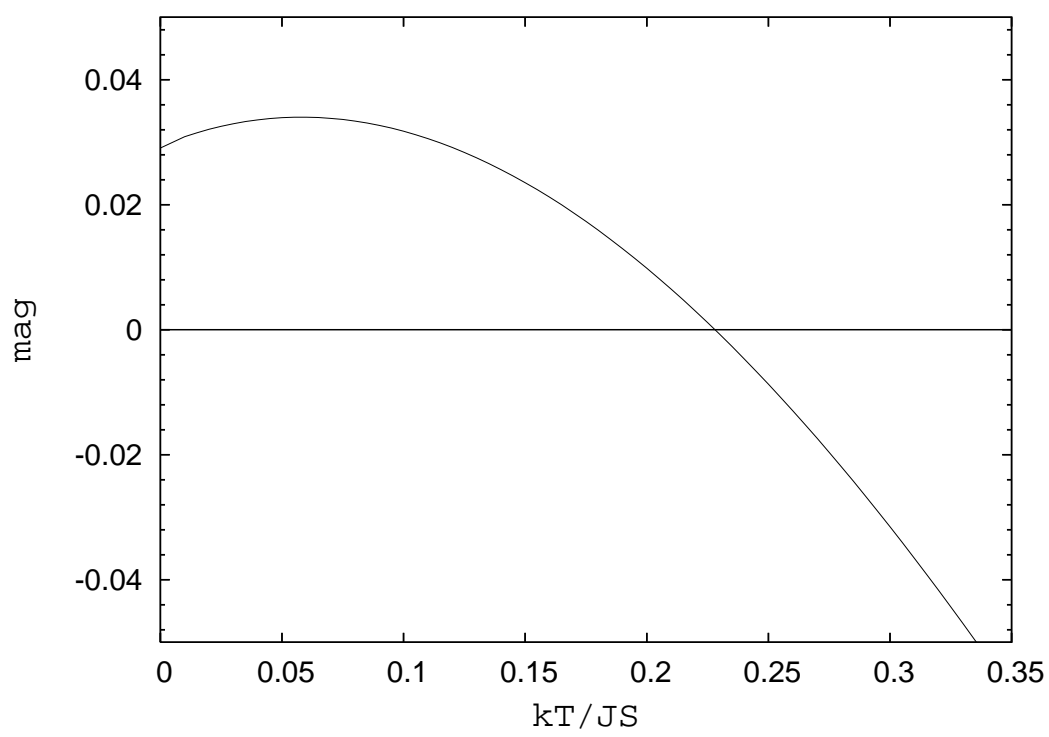

FIG. 3: Surface magnetization as a function of $k_{B} T / J S$ To model the surface we take $J_{0}=0$

[14] C. Prados et al., J. Phys.:Condens. Matter 14, 10063 (2002).

[15] Kentaro Takano, R.H. Kodama, and A.E. Berkowitz, Phys. Rev. Lett. 79, 1130 (1997). 